\title{
Pioneros de los agujeros NEGROS DESPEJARON DUDAS DE EINSTEIN
}

Pioneers of black holes clear Einstein's doubts

Juvenal Castromonte-Salinas ${ }^{1}$

Resumen

En el presente artículo se describe, cómo están relacionados el trabajo de un teórico Roger Penrose, y dos "observadores", Reinhard Genzel y Andrea Ghez, para escudriñar en el centro de la Vía Láctea un "objeto supermasivo", conocido ahora como un "agujero negro".

Palabras claves: Premio Nobel física, agujero negro, Vía Láctea.

Abstract

This article describes, how the work of Roger Penrose, a theorical physicist, and two "observers", Reinhard Genzel and Andrea Ghez, are related to scrutinized in the center of the Milky Way a

"supermassive object", now known as a "black hole".

Keywords: Physical Nobel Prize, Black Hole, Milky Way.

premio Nobel de Física de este año es bastante sorprendente. Entre los años 2017- 2020 la astrofísica obtuvo su tercer premio Nobel; así, en el 2017 fue por la detección de las ondas gravitacionales; en el año 2019, por los avances de la cosmología y el descubrimiento de exoplanetas; y, en el 2020, por los agujeros negros.

1 Profesor principal. Departamento de Ciencias Exactas, Universidad Peruana Cayetano Heredia, Lima, Perú.

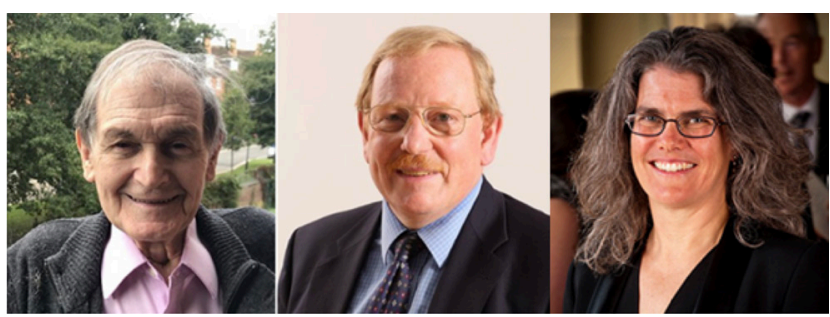

De izq. a der. Roger Penrose, Reinhard Genzel y Andrea Ghez.

El premio 2020 se dividio de la manera siguiente: una mitad le correspondió al físico teórico Roger Penrose y la otra mitad, en partes iguales, a Reinhard Genzel y Andrea Ghez. En la declaración del Comité Nobel, Penrose fue galardonado por el "descubrimiento sobre exacta predicción de la teoría general de relatividad acerca de la formación los agujeros negros"; en tanto, Genzel y Ghez por el "descubrimiento de un objeto supercompacto en el centro de nuestra galaxia". En el presente artículo se describe, cómo están relacionados el trabajo de un teórico y dos "observadores" para "ver" en el centro de la Vía Láctea un "objeto supermasivo". 
Se debe tener en cuenta que los trabajos por las que se concede los premios son bastante antíguos. Roger Penrose obtuvo sus resultados acerca de los agujeros negros en el año 1965. Los trabajos de los otros dos laureados se realizarón en los años 1990’s. En estas investigaciones se describieron las predicciones teóricas de los agujeros negros y detalles de la observación directa de objetos celestes, con todas sus propiedades y características que, originalmente, eran solo objetos hipotéticos.

\section{Orígenes de la IDEA DE los AgUJeros NegROS}

En general, la idea sobre la existencia de objetos muy masivos que tuviesen la suficiente fuerza para atrapar a la luz es bastante antigua. Ya en el siglo XVIII, esta idea la manifestaron el inglés John Michell y el francés Pierre-Simon Laplace. Sin embargo, ambos basaban sus ideas en las leyes de Newton, que muchas décadas mas tarde se entendió que ella era una teoría insuficiente para describir el comportamiento de la luz en regiones cercanas a cuerpos super masivos. La teoría capaz de poder explicar este fenómeno recién aparece en 1915. Esta es la conocida "teoría general de la relatividad" de Einstein que, actualmente, es el fundamento de la teoría de gravitación utilizada para describir los objetos celestes, incluso todo el Universo.

Las soluciones encontradas a las ecuaciones de Einstein son solo de casos particulares. En 1916, Karl Schwarzschild encontró una solución matemática, esta es acerca del agujero negro, pero para el modelo mas simple.

En los años siguientes, el problema de los agujeros negros no resultaba lo suficientemente atractivo para la comunidad de físicos, a pesar de que se habían obtenido algunos resultados interesantes. Sin embargo, la debilidad de todos estos resultados radicaba en que solo respondían a casos imaginarios y los más simples, razón por lo que muchos no consideraban serio la existencia y formación de los agujeros negros.

A mediados de la década de los 1960's, Roger Penrose se sintió atraído por los trabajos de John Wheeler acerca de los agujeros negros y las dificultades matemáticas que presentaban para su descripción.

\section{LOS AGUJEROS NEGROS, DE HIPÓTESIS A REALIDAD}

A inicios de los años 1960's se descubrieron los cuásares. El brillo de los cuásares, de acuerdo con los astrónomos, superaba el brillo de miles de galaxias, incluso a pesar de no ser visibles a simple vista, por encontrarse muy alejados de la Tierra, cerca de los límites del universo visible. Sin embargo, estaba claro que un objeto tan brillante como el cuásar no podía ser una estrella común. La razón es que cuanto mas brillo tiene una estrella, tanto más corto es su tiempo de vida. Una estrella grande y brillante rápidamente consume su hidrógeno necesario para las reacciones termonucleares.

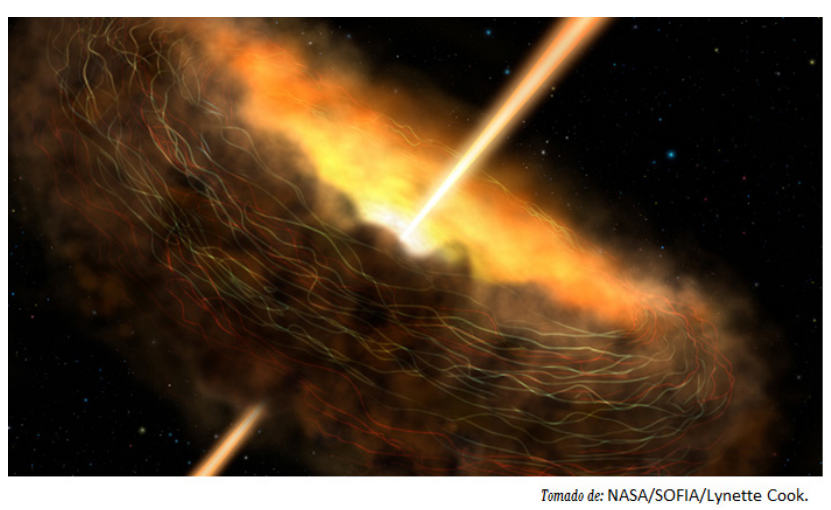

Encontrar "vivo" una estrella con el brillo de un cuásar era imposible, por lo que, para explicar su brillo, se propuso la hipótesis de que los cuásares eran agujeros negros de masas muy grandes. Estos, al atrapar a los 
objetos de su entorno, deberían irradiar parte de la energía de estos objetos "presos" como flujos brillantes.

Gracias a esta hipótesis, los físicos volvieron al problema de la rigurosidad matemática para explicar la posibilidad de la formación de agujeros negros. Fue, precisamente, Roger Penrose quien solucionó este problema, aunque en su artículo nunca mencionó ni hizo uso del término "agujero negro", que fue introducido, más tarde, por Wheeler. Para elló utilizo un formalismo matemático bastante riguroso y novedoso, para mostrar que las ecuaciones de la teoría general de relatividad de Einstein, entre sus soluciones, contenían la formación de los agujeros negros, incluso a condiciones muy alejadas de las condiciones ideales. Esta demostración, además el haber sido observados los cuásares, convenció a la comunidad de astrofísicos sobre la existencia real de los agujeros negros, hasta entonces hipotéticos, objetos exóticos, realidad que fue confirmada nuevamente en 1916, con la detección de las ondas gravitacionales.

\section{LA BÚSQUEDA DE LOS AGUJEROS NEGROS}

Casi inmediatamente al descubrimiento de los cuásares, se propuso la hipótesis de que los agujeros negros existen no solo en galaxias lejanas sino en el centro de casí todas las galaxias, incluyendo a la Vía Láctea. La masa de estos agujeros negros no es tan grande como la de los cuásares, ellos no atrapan a los objetos de su entorno con velocidades grandes, lo que no permite verlas directamente porque su emisión de energía es insuficiente.

Hasta no hace mucho, la resolución de los telescopios existentes era insuficiente para poder observar en zonas cercanas a los agujeros negros grandes. Recién con la implementación del sistema de telescopios Event Horizon
Telescope, en el año 2019, se pudo obtener la imagen de la "sombra de un agujero negro".

Los grupos de Genzel y Ghez descubrieron un agujero negro en la Vía Láctea utilizando métodos indirectos pero confiables. Ellos decidieron medir la velocidad del movimiento de las estrellas que giran a una distancia no muy grande del centro de nuestra galaxia. Este centro, por razones históricas, los astrónomos la conocen como Zagitario A* Si en el centro se tiene un objeto con masa relativamente no muy grande, entonces la velocidad de las estrellas que giran a su rededor, debe ser menor cuanto mas alejados del centro. Esto es consecuencia de las leyes de Kepler, que intuitivamente podemos hacer un paralelo con los planetas que giran alrededor del Sol, donde las velocidades de los planetas disminuyen a medida que se mueven del centro hacia la periferie. Si en el centro de nuestra galaxia no se tuviese un objeto compacto y toda la masa de la materia estuviese distribuida en un volumen grande, entonces la velocidad de las estrellas, en función de la distancia, variaría de manera diferente a la que conocemos.

La primera dificultad para este tipo de observaciones se debe a que en el centro de la galaxia se encuentran muchas estrellas y, además, gases y polvos interestelares. Es decir, este centro es poco visible. Para solucionar este inconveniente se propuso hacer observaciones utilizando luz infrarroja en lugar de luz visible debido a que las ondas infrarrojas son débilmente absorbidas por la materia interestelar. Lamentablemente, estas ondas se absorben por la atmósfera, lo que adiciona otra dificultad para las observaciones.

Otro problema era que las estrellas en el espacio celeste se mueven lentamente, para poder medir sus velocidades se necesita observarlas mucho tiempo. Es poco práctico realizar estas 
observaciones con ayuda de los telescopios espaciales, como el Hubble, que, además, realizan otras observaciones que también requieren de tiempo. Estas dificultades obligaron a utilizar teslecopios instalados en la Tierra.

Pero, con los telescopios en la Tierra se tiene una dificultad adicional, la atmósfera de la Tierra. Cuando los astónomos tratan de mirar detalles pequeños en el cielo se topan con grandes deformaciones, incluso con pequeñas oscilaciones del aire. Precisamente, la solución técnica de este inconveniente llevó a los grupos de Genzel y Ghez al descubrimiento que les permitio obtener el premio Nobel.

\section{Observando un agujero NEGRo EN El CENTRO DE NUESTRA GALAXIA}

Primero, para obtener imágenes nítides del agujero supermasivo en el centro de la Vía Láctea, Genzel y Ghez utilizaron pequeños tiempos de exposición, abrían el objetivo de la cámara un tiempo menor a un segundo. En este intervalo de tiempo, a la cámara llegaba una cantidad pequeña de luz, lo que obligo a construir, de manera ad hoc, un detector ultrasensible.

Segundo, debido al movimiento del aire, las imágenes de las estrellas en tomas diferentes se encuentran desplazadas una toma con respecto a la otra, lo que motivo a los astrónomos a utilizar un método especialmente desarrollado para reestablecer la posición inicial.

Y, finalmente, ambos grupos hicieron sus mediciones por varios años. Genzel, los realizó en el observatorio de La Silla, en el desierto de Atacama en Chile, mientras que Ghez lo hizo en el observatorio de Mauna Kea en Hawai. Mas tarde, ellos compararon sus resultados y encontraron muy buena coincidencia de ellos.
La velocidad de las estrellas, casi de manera ideal, concuerdan con las leyes de Kepler, lo que demostraba la presencia, en el centro de la galaxia, de un objeto compacto de masa muy grande.

Observaciones posteriores, realizadas con métodos mas avanzados, solo confirmaron las primeras conclusiones, mas aún, permitieron obtener argumentos más sólidos para sustentar que estos objetos masivos son efectivamente agujeros negros.

En 1992 se descubrió la estrella S2, que realiza una vuelta completa alrededor del centro en solo 16 años. Es decir, mientras se observaba el centro de nuestra galaxia, la estrella S2 ya había dado más de una vuelta, cuyo movimiento no podía ser descrito por las leyes de Newton;

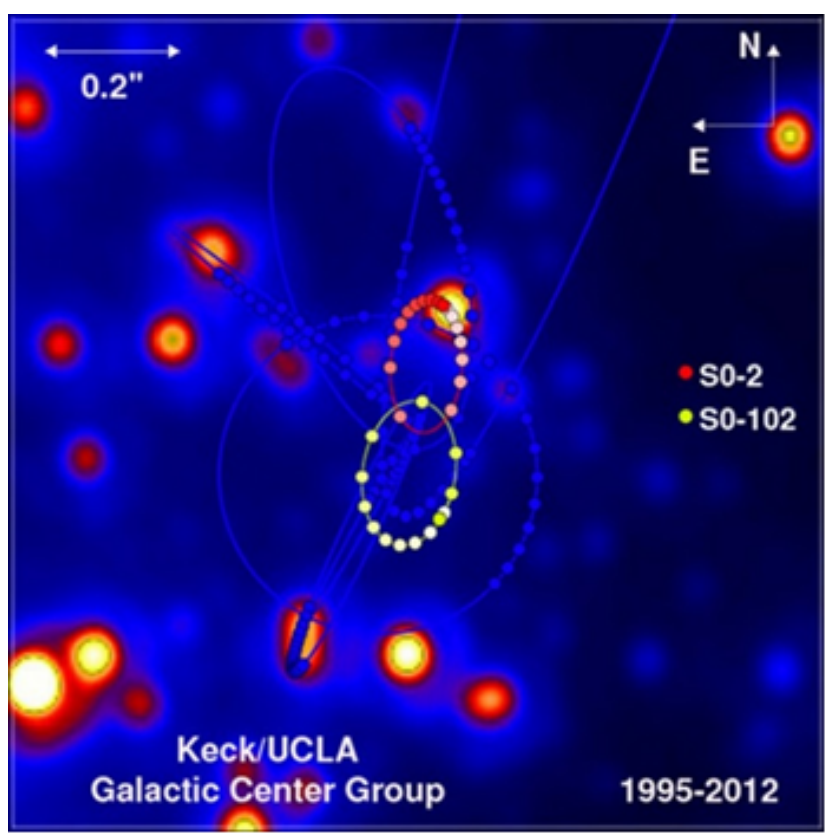

Orbitas de las estrellas S2 (codificada como S0-2) y S0-102 alrededor de un agujero negro en el centro de la Vía Láctea. Estas dos estrellas son las que se mueven mas cercanos (de los que se conocen) al agujero negro. También se muestran las órbitas de algunas estrellas del conjunto S. Cada circulo es una observación individual, obtenida entre 1995 y 2012. El agujero negro se encuentra en la región de intersección de las órbitas de las dos estrellas (tomada del site arstechnica.com) 
sin embargo, coincidia muy bien con la teoría general de la relatividad, que tomaba en cuenta la presencia de un objeto ultracompacto en el centro de la galaxia.

En el año 2019, con la ayuda de una red de telescopios, los astrónomos observaron zonas cercanas a sagitario $A^{*}$ y mostraron que sus imágenes coincidían con lo esperado de las observaciones de agujeros negros con hipermasas.

Los agujeros negros, son objetos bastante exóticos en el universo. Son objetos donde se tienen situaciones en las que las leyes de la física actual no "funcionan", para lo cuál se requiere la creación de una nueva teoría que unifique las leyes del micromundo, la física cuántica y las leyes de la gravitación que describe el mundo de las estrellas.

Los resultados obtenidos para agujeros negros nos han impuesto nuevos retos en la búsqueda de una nueva física capaz de revelarnos que otras cosas nos esconde la naturaleza.

\section{Correspondencia}

Juvenal Castromonte Salinas

Juvenal.Castromonte@upch.pe

Fecha de recepción: 25-01-2021.

Fecha de aceptación: 22-02-2021.

Conflicto de interés: ninguno, según el autor. Financiamineto: por el autor. 